\title{
Eduardo VIVEIROS DE CASTRO, Politique des multiplicités. Pierre Clastres face à l'État
}

Lauriane Guillout

\section{OpenEdition}

1 Journals

\section{Édition électronique}

URL : http://journals.openedition.org/ress/6643

DOI : $10.4000 /$ ress.6643

ISBN : $1663-4446$

ISSN : $1663-4446$

Éditeur

Librairie Droz

Édition imprimée

Date de publication : 1 décembre 2020

Pagination : 257-260

ISSN : 0048-8046

\section{Référence électronique}

Lauriane Guillout, «Eduardo VIVEIROS DE CASTRO, Politique des multiplicités. Pierre Clastres face à l'État », Revue européenne des sciences sociales [En ligne], 58-2 | 2020, mis en ligne le 01 décembre 2020, consulté le 03 décembre 2020. URL : http://journals.openedition.org/ress/6643 ; DOI : https:// doi.org/10.4000/ress.6643

Ce document a été généré automatiquement le 3 décembre 2020.

(c) Librairie Droz 


\title{
Eduardo VIVEIROS DE CASTRO, Politique des multiplicités. Pierre Clastres face à l'État
}

\author{
Lauriane Guillout
}

\section{RÉFÉRENCE}

Eduardo VIVEIROS DE CASTRO, 2019, Politique des multiplicités. Pierre Clastres face à l'État, postface et traduction de Julien Pallotta, Bellevaux, Éditions Dehors, $160 \mathrm{p}$.

1 L'anthropologue brésilien Eduardo Viveiros de Castro compte parmi les principales figures du renouveau de l'ethnologie américaniste. Il a initié ce qu'il est convenu d'appeler le tournant ontologique en anthropologie avec ses concepts de multinaturalisme et de perspectivisme qui soumettent à la critique le dualisme entre nature et culture, incapable de décrire les cosmologies amazoniennes. Encore peu traduits, les travaux de Viveiros de Castro attestent pourtant du lien qui s'est noué, depuis l'avènement $\mathrm{du}$ structuralisme, entre l'anthropologie sud-américaine et française. Les innovations conceptuelles de l'anthropologue brésilien sont, en effet, le fruit de son dialogue continu avec Claude Lévi-Strauss et son élève trublion, Pierre Clastres, dont il n'a cessé de retravailler le legs en héritier critique. Il rencontrait déjà les recherches de Clastres sur le prophétisme des Tupi-Guarani dans son travail de thèse consacré aux Arawaté du Nord du Brésil. Attentif aux phénomènes de prédation au sein des sociétés amérindiennes, Viveiros de Castro s'est également intéressé aux études clastriennes sur la guerre. Politique des multiplicités - traduit du portugais, postface de la réédition de 2011 du recueil d'articles posthumes Arqueologia da violência, São Paulo, Cosac \& Naify, 1980 (recueil originairement paru aux Éditions du Seuil, la même année 1980, sous le titre de Recherches d'anthropologie politique, réédité en 2012) perpétue ainsi un compagnonnage théorique que n'explique pas la seule proximité des terrains ethnographiques. C'est, plus profondément, une affinité de style et de pensée qui réunit les deux anthropologues. Aucun ne dissocie l'écriture et la pratique de 
l'anthropologie d'un engagement envers ceux que Clastres, et à sa suite Viveiros de Castro, nomment les indiens. À rebours d'une conception positiviste de la discipline véhiculée par un certain structuralisme, chacun a réaffirmé l'implication mutuelle de l'anthropologie et de la philosophie avec pour ambition de les transformer ensemble plutôt que de choisir entre elles. Clastres fut l'un des premiers à relancer cette communication en s'écartant de la méthode structurale qui échouait, selon lui, à rendre compte de l'inventivité politique propre à toute société. Ce dialogue post-structuraliste entre philosophie et anthropologie trouve aujourd'hui son plein déploiement dans l'œuvre de Viveiros de Castro.

2 Mais s'il a des adeptes, ce style a aussi de nombreux détracteurs. L'anthropologie française a vu dans la notion clastrienne de «société contre l'État » une hypostase qui trahit l'anarchisme de l'ethnologue sans rendre compte de la singularité des sociétés qu'il étudie. Dans son immense majorité, elle a fait valoir que cette proposition s'annule d'elle-même par son anachronisme. Comment les sociétés amérindiennes auraient-elle pu s'instituer politiquement contre l'avènement d'un pouvoir étatique supposé leur être ultérieur? Comble du paradoxe, Clastres aurait reconduit les présupposés ethnocentristes de la philosophie occidentale en cédant à cette illusion rétrospective. Elle l'aurait détourné de son ambition initiale : démontrer la contingence historique de la forme étatique afin de restituer l'originalité politique des collectifs amérindiens. En effet, soutenir qu'ils s'organisent pour conjurer la transcendance du pouvoir reviendrait à maintenir ces collectifs sous la dépendance d'un État, pensé comme l'horizon nécessaire du politique. Ainsi, le concept qui a rendu le travail de Clastres célèbre l'a également fait tomber en désuétude. S'il a suscité l'intérêt des philosophes l'écho qu'il eut chez Claude Lefort, Miguel Abensour, ou Gilles Deleuze en atteste -, il a été rangé par les ethnologues français dans le cabinet des curiosités anthropologiques. Toute autre a été la réception de Clastres au Brésil où sa thèse a joué, comme l'enseigne Viveiros de Castro, un rôle moteur dans la rénovation de l'ethnologie amazonienne.

3 Pour l'anthropologue brésilien, on ne peut disqualifier le concept de société contre l'État au motif des inclinations idéologiques de son auteur. "La relation entre l'anarchisme de Clastres et sa spécialisation dans les sociétés des basses terres de l'Amérique du Sud» (p. 21) atteste plutôt que la pratique ethnographique de Clastres repose sur une dynamique d'échange et de co-constitution des points de vue entre l'observateur et l'observé. Loin d'avoir projeté sur les collectifs amazoniens une théorie politique qui leur était étrangère, il a su amorcer un processus de traduction de leur altérité et de leur inventivité politiques. La société contre l'État n'est donc pas un " artefact» construit à partir d'utopies libertaires toutes occidentales, mais une « redescription abstraite et (re)stylisée [...] de cosmopratiques historiquement et culturellement situées » (ibid.). Il serait donc plus juste de dire que «l'anarchisme » de l'ethnologue est celui des indiens Chulupi, M'bya et Guayaki-Aché auprès desquels il a séjourné. Retournant contre elles-mêmes les critiques habituelles d'anachronisme et d'ethnocentrisme, Viveiros de Castro réaffirme ainsi la pertinence ethnographique du concept de société contre l'État. Mais cette prise de position ne doit pas tromper. Elle ne consiste pas en une défense aveugle venant éliminer les équivoques du concept, mais en une réappropriation critique qui refuse de « détourner pudiquement le regard de ses hyperboles dérangeantes, de ses impatiences et imprécisions » (p. 30). Il s'agit, pour Viveiros de Castro, de reprendre «les intuitions anthropologiques fulgurantes » 
(ibid.) ramassées dans la thèse de la société contre l'État pour en redécouvrir la charge intempestive.

4 Cette réappropriation critique suit deux voies principales qui infléchissent et prolongent les intuitions clastriennes. La première approfondit la description politique de la société contre l'État en y incluant ces autres « citoyens » que sont, en Amazonie, les non-humains. Sur ce point, Clastres n'avait soufflé mot. Ses affinités avec l'humanisme des phénoménologues et l'influence du sociocentrisme de Durkheim l'ont, pour Viveiros de Castro, empêché de concevoir une constitution politique multispécifique qui ne se limite pas aux seuls humains. «Il restait à politiser la nature, ou le cosmos - penser la dimension cosmopolitique de la société contre l'État» (p. 104). Politique des multiplicité ouvre ce chantier en intégrant aux énoncés originels de Clastres l'idée d'un multinaturalisme ontologique. Il ressort de cette inflexion que les sociétés amérindiennes s'instituent politiquement contre l'État pour la même raison qu'elles organisent leur vie collective sans considérer nécessaire l'extériorisation de ce que les modernes nomment nature. "C'est une telle non séparation cosmologique [...] qui devrait être mise en parallèle avec la non-séparation politique qui définit la société contre l'État» (p. 106). En d'autres termes, une autorité politique transcendante a d'autant moins la possibilité de s'installer lorsque les dimensions sociales et écologiques de la vie collective, plutôt que distinguées, prennent la forme d'une multiplicité de rapports entre des acteurs humains et non humains, tous dotés d'intentionnalité. Un monde où prévaut une telle "dissémination de l'agentivité "subjective" " ne peut faire place à « un point de vue politique unifiant, occupé par un Agent (l'agent de l'Un) qui contiendrait en soi le principe de l'humanité et de la socialité »(p. 109).

5 La seconde voie, qu'emprunte la réappropriation de ce concept, est plus inattendue. D'inspiration deleuzienne, elle subvertit de l'intérieur la typologie binaire et rigide arrêtée par Clastres. Il distinguait seulement, pour les opposer radicalement, deux formes de société : sociétés modernes, «à État », d’un côté ; sociétés sauvages, « contre l'État », de l'autre. Or, à l'inverse d'un type exprimant une singularité strictement non occidentale, Viveiros de Castro conçoit la société contre l'État comme une potentialité universelle, susceptible d'être actualisée hors de contextes ethno-historiques prédéfinis. "Société-contre-l'État, en somme, est un concept qui désigne un régime d'intensité ou un fonctionnement virtuel omniprésent » (p. 90). Puisque «si l'État a toujours existé, comme l'ont soutenu Deleuze et Guattari, alors la société primitive existera toujours : comme extérieur immanent à l'État, force d'antiproduction toujours menaçant les forces productives, multiplicité non intériorisable par les grandes machines mondiales » (p. 27-90). Ainsi comprise, la société contre l'État ne donne aucun critère stable pour classer les diverses formations sociales puisqu'elle constitue plutôt une différenciation interne à chaque d'elle. Susceptible d'être activée par tous collectifs, quand bien même elle n'a pas la permanence de l'institution comme dans les basses terres d'Amazonie, la société contre l'État s'avère un possible universel.

6 Cette redéfinition peut surprendre. N'est-ce pas paradoxalement rabattre l'autre sur le même, et finalement déprécier la particularité des cosmopolitiques amazoniennes, que de conférer une telle omniprésence à la société contre l'État ? Pour Viveiros de Castro, l'universalité du concept n'annule pas les différences. Au contraire, elle fait jouer à plein l'épreuve réflexive de l'altérité en démultipliant plus encore ses images. Cette universalisation prolonge et approfondit donc «la révolution copernicienne " que 
Clastres appelait de ses voeux. Car, il ne suffit pas d'opposer les sociétés à et contre l'État pour troubler les a priori de la philosophie politique occidentale et modifier ses attendus. Il faut encore que la troublante altérité politique des indiens devienne un peu la nôtre, en venant «nous renvoyer une image de nous-mêmes dans laquelle nous ne nous reconnaissons pas » (p. 27). Alors, seulement, les leçons politiques de l'ethnologue - donc, celles des peuples qu'il étudie - peuvent être prises au sérieux. L'épreuve réflexive de l'altérité débute vraiment lorsque la philosophie politique, à partir des enseignements de l'anthropologie, ne se contente pas de changer son regard sur lesdits " primitifs », mais modifie aussi, en retour, l'idée qu'elle se fait des modernes.

$\mathrm{Au}$ terme de cette transfiguration de la thèse clastrienne, nos sociétés n'ont plus seulement à admettre l'existence d'authentiques "politiques sauvages » ne manquant de rien bien qu'elles ne s'ordonnent pas sous l'égide de l'État. Plus fortement, nos sociétés se découvrent travaillées par un «devenir-indien du politique » qui prend toute sa consistance avec les bouleversements écologiques actuels. À l'ère de l'anthropocène, en effet, "nous sommes tous susceptibles d'être confrontés, à un moment ou à un autre, en réalité à chaque moment, à devoir choisir entre "devenirindien" - habiter les marges, vivre aux limites de la clôture (il n'est pas nécessaire d'aller dormir dans les bois pour cela, c'est d'autre chose qu'il s'agit) - ou demeurer dans les centres fortifiés » (p. 63). Il est possible de considérer que les mouvements qui s'attachent désormais à la terre comme à un objet de revendications de droits - contre les exploitations qu'elle subit -, activent quelque chose de la société contre l'État. Partout où s'inventent des modalités alternatives de la coexistence entre humains et non humains, se manifeste «l'altérité intensive » que désigne la société contre l'État. Viveiros de Castro ne pouvait donc pas nous éloigner davantage des critiques traditionnelles de la thèse clastrienne. Pertinente pour appréhender le paysage cosmopolitique amérindien, elle l'est tout autant pour décrire les lieux où les transformations écologiques de la politique des modernes prend le plus d'intensité. À la question qu'il posait, il faut donc répondre positivement : «À l'heure actuelle alors que penser est devenu la tâche de la plus grande urgence et de la plus extrême gravité - qui ne s'est pas encore rendu compte que "notre civilisation", certainement, et que notre espèce, fort probablement, sont entrées dans une crise aux dimensions absolument inouïes, et que cette crise est en train de détruire une bonne partie de la vie non humaine de la planète? - à l'heure actuelle, nous demandions nous, l'excentrique ethnologue et son étrange société primitive sont-ils toujours bons à penser ? (p. 66).

\section{AUTEURS}

\section{LAURIANE GUILLOUT}

Université de Bruxelles, СTP 